\title{
Travail des femmes et fécondité dans les pays en développement : que nous a appris l'Enquête mondiale de la fécondité?
}

FEMALE LABOR AND FERTILITY IN DEVELOPING COUNTRIES: WHAT DOES THE WORLD FERTILITY SURVEY TELL US?

EL TRABAJO DE LAS MUJERES Y LA FECUNDIDAD EN LOS PAISES EN DESAROLLO : QUE NOS HA ENSENADO LA ENCUESTA MUNDLAL DE LA FECUNDIDAD?

Jean Poirier, Victor Piché et Ghyslaine Neill

Volume 18, numéro 1, printemps 1989

Démographie et femmes

URI : https://id.erudit.org/iderudit/010006ar

DOI : https://doi.org/10.7202/010006ar

Aller au sommaire du numéro

Éditeur(s)

Association des démographes du Québec

ISSN

0380-1721 (imprimé)

1705-1495 (numérique)

Découvrir la revue

Citer cet article

Poirier, J., Piché, V. \& Neill, G. (1989). Travail des femmes et fécondité dans les pays en développement : que nous a appris l'Enquête mondiale de la fécondité? Cahiers québécois de démographie, 18(1), 159-184.

https://doi.org/10.7202/010006ar
Résumé de l'article

L'objectif de cet article est d'examiner dans quelle mesure les données de l'Enquête mondiale de la fécondité (EMF) sont adéquates pour l'analyse de la relation entre le travail des femmes et la fécondité. Après avoir passé en revue les objectifs généraux de l'EMF, les auteurs présentent les hypothèses théoriques et les questions par lesquelles on a tenté de vérifier celles-ci. Une troisième partie est consacrée aux principales analyses comparatives réalisées, à leurs résultats fondamentaux et aux diverses interprétations qui en ont été dégagées. Les auteurs concluent en faisant ressortir en quoi ces données de l'EMF sont effectivement utiles, à la condition que l'on tienne compte du contexte institutionnel global de chaque société.
Tous droits réservés @ Association des démographes du Québec, 1989
Ce document est protégé par la loi sur le droit d'auteur. L'utilisation des services d'Érudit (y compris la reproduction) est assujettie à sa politique d'utilisation que vous pouvez consulter en ligne.

https://apropos.erudit.org/fr/usagers/politique-dutilisation/ 
Cahiers québécois de démographie

Vol. 18, no 1, printemps 1989

\title{
Travail des femmes et fécondité dans les pays en développement : que nous a appris l'Enquête mondiale de la fécondité ?
}

\author{
Jean POIRIER, Victor PICHÉ et Ghyslaine NEILL*
}

\section{INTRODUCTION}

Les principales formulations théoriques visant à comprendre et à expliquer les mouvements de la fécondité attribuent un rôle important au travail des femmes, et plus particulièrement à ses caractéristiques et aux transformations qu'il subit. L'abondance des travaux qui ont en effet tenté d'expliquer les liens entre fécondité et activité en témoigne largernent1. Dans cette perspective, on comprend que le programme de l'Enquête mondiale de la fécondité (EMF), qui constitue la tentative la plus ambitieuse pour mesurer les comportements en matière de fécondité et en comprendre les déterminants, ait fait une large place aux questions concernant le travail des femmes. Ce sont d'ailleurs les seules questions qui possèdent un fondement théorique explicite dans l'Enquête.

* Département de démographie de l'Université de Montréal. Ceet article se situe dans le cadre d'un projet de recherche plus vaste qui vise à analyser le travail des femmes dans la Caraïbe en jumelant des données quantitatives et qualitatives. Ce projet s'effectue en collaboration avec Huguette Dagenais (codirectrice) du département d'anthropologie de l'Université Laval, et est financé par le Conseil de recherches en sciences humaines du Canada (CRSHC) ainsi que par le Fonds FCAR. Nous voudrions souligner la contribution de Joël W. Gregory, décédé le 29 juillet 1988, à l'élaboration et à la codirection des premières étapes du projet.

1. Pour une revue critique des diverses approches théoriques, voir Kempeneers (1985) pour les pays développés, et Standing (1983) pour les pays en développement. 
A l'heure où l'on dresse le bilan de cette opération de collecte et d'analyse de données 2 , il nous semble opportun de s'interroger sur ce que nous a appris l'EMF en ce qui concerne les liens entre le travail des femmes et la fécondité dans les pays en développement. Certes, on peut penser avec Davis (1987) que la contribution de ce qu'il appelle "the world's most expensive survey" ne valait pas les 40 millions de dollars (des États-Unis) investis, compte tenu de l'absence de réflexion théorique préalable3. Mais étant donné la formulation par l'EMF d'hypothèses explicites sur les liens entre emploi féminin et fécondité, on peut se demander dans quelle mesure le jugement global de Davis s'applique à cette dimension particulière de l'enquête. Par ailleurs, à partir de résultats contradictoires, certains auteurs comme Cleland en arrivent à remettre en question la pertinence même de l'hypothèse d'un lien entre emploi et fécondité. Cette dernière interprétation est d'autant plus surprenante que l'EMF avait fait un effort particulier pour proposer une définition du travail plus large que celle utilisée dans les recensements ou même dans la plupart des enquêtes sur l'emploi. On en vient ainsi à se demander si l'on ne doit pas carrément écarter les données de l'EMF dans l'étude des liens entre le travail des femmes et la fécondité.

C'est la question qui fera l'objet de cet article. Pour y répondre, nous passerons d'abord en revue les objectifs généraux du programme de l'EMF, le contexte dans lequel ces objectifs s'inscrivent ainsi que les choix méthodologiques qu'ils sous-tendent. En second lieu, nous examinerons les hypothèses théoriques relatives au lien entre le travail des femmes et leur fécondité, de même que les questions par lesquelles on a tenté de vérifier ces hypothèses. Une troisième partie sera consacrée aux principales analyses comparatives réalisées, à leurs résultats fondamentaux ainsi qu'aux interprétations variées qu'ils ont reçues. Après avoir dressé notre propre bilan du programme de l'EMF concernant les liens entre travail des femmes et fécondité dans les pays en développement, nous discuterons enfin de l'utilité des données ainsi collectées dans le contexte de l'émergence de problématiques élargies des liens entre travail des femmes et démographie dans les pays en

2. Voir Cleland et Hobcraft (1985), Cleland et Scott (1987), et Davis (1987).

3. "En l'absence de cadre théorique permettant de fonder les questions posées, toute la méthodologie d'enquête disponible de par le monde ne produira aucune lumière" (Davis, 1987 : 831; notre traduction). 
développement ${ }^{4}$. Nous ferons ainsi ressortir en quoi ces données sont effectivement utiles, mais à la condition que les liens entre travail et fécondité soient analysés en tenant compte du contexte institutionnel global de chaque société, ce qui implique le recours à d'autres sources d'informations.

\section{LE PROGRAMME DE L'ENQUETE MONDIALE DE LA FÉCONDITÉ : UN BREF HISTORIQUE}

Au début des années soixante-dix, plusieurs gouvernements et institutions internationales étaient vivement préoccupés par les conséquences économiques et sociales engendrées par les forts taux d'accroissement de la population que connaissent les pays en développement. C'est dans ce contexte que le projet du programme de l'EMF a été mis sur pied5. Ce programme a été financé en majeure partie par le Fonds des Nations Unies pour la population (FNUAP) et l'Agence américaine pour l'aide au développenent (USAID)6, son exécution étant confiée à l'International Statistical Institute (ISI) en étroite collaboration avec l'Union internationale pour l'étude scientifique de la population (UIESP). Au total, de 1974 à 1982, 42 pays en développement ont participé au programme de l'EMF?

4. Pour une discussion de ces diverses problématiques, voir Neill (1988).

5. World Fertility Survey (WFS) en anglais.

6. Un support financier additionnel a été assuré par les gouvernements du Royaume-Uni, de la France, du Canada et du Japon.

7. Notons que 20 pays développés ont également participé au programme de l'EMF, mais sans assistance financière et technique. 
Dans sa formulation officielle, l'objectif du programme est défini ainsi : "L'Enquête mondiale de la fécondité (EMF) est un programme international de recherche dont le but est d'évaluer l'état actuel de la fécondité humaine dans le monde. Afin d'atteindre cet objectif, des enquêtes par sondage sur la fécondité sont mises en oeuvre et financées dans le plus grand nombre de pays possible. Ces études élaborées et réalisées de façon scientifique fournissent des données représentatives au niveau national et comparables au niveau international" (WFS, 1976). A cet objectif général s'en ajoutent trois d'ordre scientifique : la mesure des comportements en matière de fécondité, la compréhension de leurs déterminants et la formulation de politiques pertinentes visant à influencer ces comportements. Comme le soulignent Cleland et alii (1987), chacun de ces objectifs a tour à tour été considéré comme prioritaire, aucune hiérarchie n'ayant été clairement établie.

Pour atteindre ces objectifs, les enquêtes ont été réalisées à partir d'un même questionnaire de base. L'utilisation de ce questionnaire est d'ailleurs obligatoire pour qu'un pays puisse participer au programme. Pour justifier cette exigence, on a évoqué deux types de considérations. Premièrement, d'un point de vue pratique, l'adoption d'un questionnaire de base uniforme facilite la gestion et l'assistance technique dans un contexte où la majorité des pays participants ne disposent pas des ressources humaines et matérielles nécessaires pour mener de telles enquêtes. En second lieu, d'un point de vue plus théorique, il faut assurer la comparabilité des données au niveau international. Soulignons que cette exigence s'inscrivait dans la vogue que connaissait l'analyse comparative des sociétés à la fin des années soixante et au début des années soixante-dix, telle qu'exemplifiée par les travaux de Parsons (1966). L'approche dominante en démographie sociale considérait alors que tous les pays en développement devraient connaître un même type de changement social, la "modernisation"8, et de ce fait devraient passer par les mêmes étapes de la "transition démographique" (Tabutin, 1984; Goldscheider, 1982). Nous reviendrons sur les implications de ce contexte théorique en conclusion.

8. Voir Tipps (1973) pour une excellente critique des origines de la théorie de la modernisation, des postulats qui la sous-tendent et des travaux empiriques qui s'en inspirent. 
Si le principe de l'adoption d'un questionnaire de base obligatoire renvoie au contexte scientifique de l'époque, l'élaboration de son contenu nous informe sur l'état de la discipline en ce qui concerne l'analyse de la fécondité et de ses déterminants. Westoff et Ryder, chargés d'établir les premières versions du questionnaire individuel de base, se sont largement inspirés d'un ouvrage des Nations Unies paru en 1969. Ces premières versions ont été envoyées à plus de 200 démographes de façon à recueillir leurs commentaires. Certains de ceux-ci ont fait valoir que le contenu du questionnaire devait découler d'un cadre théorique. Mais, comme le soulignèrent par la suite Cleland et alii $(1987: 39)$ :

"Ces arguments ont été neutralisés avec succès par ceux qui soulignaient l'absence d'un cadre commun et qui soutenaient qu'une approche pragmatique était plus appropriée à un projet international.

En conséquence, les questionnaires EMF sont essentiellernent a-théoriques. A la seule exception de l'emploi féminin, pour lequel le choix des questions a été influencé par le débat théorique concernant les relations entre travail et comportement reproductif, le contenu du questionnaire reflète la nature descriptive traditionnelle des enquêtes démographiques plutôt que des hypothèses théoriquernent fondées" (notre traduction).

Le questionnaire individuel de base finalement adopté comprend sept sections et comporte au total 112 questions fixes et un certain nombre de questions supplémentaires, selon le nombre d'événements démographiques vécus par la répondante (voir tableau 1). Il s'adresse aux fernmes âgées de 15 à 49 ans 9.

En plus du questionnaire de base, un choix de sept modules optionnels était proposé aux pays participants pour approfondir des points particuliers. Quatre de ces modules concernent les variables intermédiaires de la fécondité (régulation de la fécondité, planification familiale, facteurs autres que la contraception affectant la fécondité, et avortement), deux autres s'intéressent aux facteurs socio-économiques (revenu familial, patrimoine des ménages et

9. Plusieurs pays ont ajouté comme critère de sélection des répondantes qu'elles devaient avoir été en union au moins une fois dans leur vie. 
coûts-bénéfices des enfants, et caractéristiques de la communauté), et un dernier module porte sur la mortalité générale. Ces modules ont été peu utilisés en raison surtout de la longueur du questionnaire de base et des délais dans leur élaboration (Singh, 1987).

\section{Tableau 1}

Contenu du questionnaire de base de l'EMF

\begin{tabular}{|c|c|c|c|}
\hline & $\begin{array}{c}\text { Sections } \\
\text { du questionnaire }\end{array}$ & $\begin{array}{l}\text { Nombre de } \\
\text { questions } \\
\text { fixes }\end{array}$ & $\begin{array}{c}\text { Nombre de } \\
\text { questions } \\
\text { supplémentaires }\end{array}$ \\
\hline 1) & $\begin{array}{l}\text { Renseignements sur } \\
\text { la répondante }\end{array}$ & 12 & $\begin{array}{l}\text { questions spéci- } \\
\text { fiques à chaque } \\
\text { pays (religion, } \\
\text { ethnicite, lan- } \\
\text { gue...) }\end{array}$ \\
\hline 2) & $\begin{array}{l}\text { Historique des } \\
\text { maternités }\end{array}$ & 22 & $\begin{array}{l}4 \text { questions pour } \\
\text { chaque naissance } \\
\text { vivante ou non- } \\
\text { vivante }\end{array}$ \\
\hline 3) & $\begin{array}{l}\text { Connaissance et pratique } \\
\text { de la contraception }\end{array}$ & 23 & - \\
\hline 4) & Historique des unions & 8 & $\begin{array}{l}4 \text { questions pour } \\
\text { chaque mariage } \\
\text { antérieur }\end{array}$ \\
\hline 5) & $\begin{array}{l}\text { Régulation de la } \\
\text { fécondité }\end{array}$ & 23 & - \\
\hline 6) & Activité professionnelle & 14 & - \\
\hline 7) & $\begin{array}{l}\text { Renseignements sur le } \\
\text { dernier conjoint }\end{array}$ & 10 & - \\
\hline
\end{tabular}

Source : Cleland et alii, $1987: 32$.

Soulignons enfin que la place prépondérante qu'occupent les questions sur la connaissance et la pratique de la contraception ainsi que sur les attitudes concernant la taille de la famille (régulation de la fécondité) dans l'EMF 
illustre bien l'héritage des enquêtes "Connaissance, attitude et pratique de la contraception" (CAP) en vogue au cours des années soixante (Miro, 1981).

Pour faire suite à cette brève mise en contexte du programme de l'EMF, nous porterons maintenant notre attention sur les liens présentés entre le travail des femmes et leur fécondité, ainsi que sur la façon dont on a cherché à les saisir.

\title{
2. TRAVAIL DES FEMMES ET FÉCONDITÉ. LES HYPO'THESES ET LEUR VÉRIFICATION
}

Comme nous l'avons déjà mentionné, la section du questionnaire individuel de base portant sur le travail de la répondante est la seule qui possède un fondement théorique explicite. En effet, dans la documentation de l'EMF sur le questionnaire de base, on donne aux questions concernant le travail de la femme la justification suivante (WFS, $1976: 16$ ) :

\begin{abstract}
"Dans toutes les sociétés et toutes les cultures, il existe une corrélation étroite entre le travail des femmes et la fécondité, notamment dans les zones urbaines. Bien qu'il soit difficile de déterminer le sens de la causalité, la notion fondamentale est que les femmes qui travaillent sont exposées au monde extérieur dont les valeurs sont théoriquement incompatibles avec les valeurs familiales.
\end{abstract}

En outre, si la femme travaille à plein temps, loin de son foyer et en tant que salariée, le temps, l'énergie et l'intérêt absorbés par son activité ne peuvent être consacrés à la mise au monde des enfants."

Cette formulation renvoie à deux approches interreliées en démographie sociale pour expliquer les comportements différentiels en matière de fécondité : celle de l'incompatibilité des rôles et celle de l'orientation en termes de rôle sexué ("sex-role orientation").

La première approche repose sur l'hypothèse que l'occupation simultanée des rôles de travailleuse et de mère engendre une situation 
conflictuelle, l'un de ces rôles devant nécessairement être "sacrifié" au profit de l'autre. Cependant, certaines conditions peuvent atténuer ces conflits. Par exemple, le travail à domicile ou sur l'exploitation familiale permettrait dans une large mesure de concilier les deux rôles. De même, la présence de parents ou domestiques pouvant assurer à faibles coûts la garde des enfants atténuerait les problèmes d'incompatibilité. Ces conditions se rencontreraient fréquemment dans les pays en voie de développement (Engracia, 1981).

La seconde approche classe les femmes en deux groupes selon leur orientation en termes de rôle de sexe : les traditionnelles et les modernes. Les premières privilégient leur rôle de mère et de conjointe, valorisant de ce fait les grandes familles. Si elles travaillent, ce sera par nécessité économique. Les modernes, au contraire, considèrent le travail des femmes comme une alternative légitime au rôle de mère. C'est un intérêt personnel qui les poussera alors sur le marché du travail. Elles auront en général une faible fécondité (Engracia, 1981). Dans cette optique, si le fait de travailler constitue un indicateur de l'orientation en termes de rôle de sexe, il contribue également à modifier cette orientation. L'hypothèse qui en découle est la suivante (Kupinsky, $1977: 214$ ) :

"... la femme qui travaille (à l'extérieur) a plus de possibilités de contact avec des attitudes rationnelles à l'égard de la dimension et de l'espacement de la descendance, et elle a plus de possibilités de connaître et utiliser des méthodes contraceptives efficaces que celle qui ne travaille pas" (notre traduction).

A partir d'une problématique qui combine ces deux approches, les questions concernant le travail des femmes dans l'EMF visent deux grands objectifs. D'une part, elles servent d'indicateurs pour mesurer le degré d'incompatibilité entre les rôles de mère et de travailleuse. Par exemple, l'occupation d'un emploi de "col blanc" à l'extérieur du foyer impliquerait un fort degré d'incompatibilité. D'autre part, on utilise ces questions pour établir plusieurs barèmes destinés à situer l'enquêtée en ce qui a trait aux valeurs traditionnelles ou modernes (WFS, $1976: 16$ ). Ainsi le travail sur la ferme familiale traduirait l'adhésion à des valeurs traditionnelles. 
Cela dit, le questionnaire de base de l'EMF utilise une définition du travail qui inclut toute activité productive autre que les "occupations ménagères". Le préambule de la section du questionnaire sur l'activité professionnelle le spécifie dans les termes suivants à l'enquêtée (WFS, 1976) :

"Comme vous le savez, beaucoup de femmes travaillent. Je veux dire, qu'elles exercent une activité en plus du travail de la maison. Certaines prennent un emploi pour lequel elles sont payées en espèces ou en nature, d'autres tiennent une boutique ou un petit commerce, ou cultivent les champs de la famille. Est-ce que vous travaillez en ce moment?"

Il convient de souligner que des 42 pays participants, seulement 26 ont utilisé cette définition. La plupart de ceux qui ne s'y sont pas conformés ont notamment occulté le travail familial non rémunéré (Cleland et alii, $1987: 74$ ).

Un premier groupe de questions concerne l'activité professionnelle exercée au moment de l'enquête ou la dernière activité exercée depuis le mariage10 : l'occupation principale (codée en neuf grandes catégories), le lieu de travail (au foyer, sur la ferme familiale ou à l'extérieur), la catégorie d'employeur (parent ou non-parent), le mode de rémunération (en argent ou en nature). On enregistre aussi la durée du travail depuis le premier mariage. La décision de ne collecter des renseignements que sur l'activité la plus récente a été prise "afin de ne pas allonger inutilement le questionnaire" (WFS, 1976 : 16) :

"Cette décision peut se justifier par le fait que la plupart des femmes n'auront pas exercé d'emploi, ou n'en auront exercé qu'un seul, depuis leur mariage. Quant à celles qui ont eu plusieurs activités professionnelles, il est permis de supposer que la plupart d'entre elles ont toujours fait le même travail".

Il faut noter que cette justification ne s'appuie sur aucune observation empirique, le questionnaire de base de l'EMF n'ayant fait l'objet d'aucun prétest sérieux.

10. Le terme "mariage" comprend les unions "illégitimes ou consensuelles" là où elles sont couramment pratiquées. 
Un second groupe de questions porte sur l'activité professionnelle avant le (premier) mariage11 : principale occupation, durée, catégorie d'employeur, mode de rémunération. On demande également si la répondante a travaillé entre son (premier) mariage et la naissance de son premier enfant.

Il importe ici de constater que ces questions ne permettent pas de repérer sur le plan chronologique les activités professionnelles des femmes ni pour la période précédant leur (premier) mariage, ni pour celle qui le suit. On dispose tout au plus du nombre total d'années pendant lesquelles les femmes ont travaillé. Le caractère flou de cette information contraste fortement avec la grande précision chronologique des renseignements de l'EMF concernant la nuptialité et surtout la fécondité.

\section{LES ANALYSES COMPARATIVES MENÉES A PARTIR DES DONNÉES DE L'EMF SUR LES LIENS ENTRE LE TRAVAIL DES FEMMES ET LA FÉCONDITÉ}

Nous avons choisi d'aborder la littérature empirique réalisée à partir des données de l'EMF sur les liens entre travail des femmes et fécondité en passant en revue les principales analyses comparatives qui ont impliqué plus de dix pays (par opposition aux études de cas dont certaines seront présentées dans la prochaine section). C'est en effet aux travaux de ce type qu'étaient destinées en priorité les données de l'EMF. Parmi ces travaux, nous avons retenu ceux qui utilisent les modèles d'analyse multivariée en raison du plus grand intérêt que présentent leurs résultats. Nous terminerons cette revue en examinant les interprétations qu'ont suggérées ces résultats.

L'article de Rodriguez et Cleland (1981) constitue certes le premier travail de ce type et le plus cité, ce qui en fait une référence obligée. Il s'agit d'une étude sur les déterminants socio-économiques de la fécondité dans 20

11. Plusieurs pays de la Caraỉbe ont choisi comme point de référence la naissance du premier enfant plutôt que le mariage. 
pays 12, à partir d'une analyse de régression multiple. Les auteurs ont utilisé comme variable dépendante la fécondité des cinq années précédant l'enquête pour les femmes ayant été en union au moins une fois dans leur vie. Les six variables indépendantes retenues sont : la résidence actuelle, le niveau de scolarité de la répondante et celui de son (dernier) conjoint, le statut de travail de la répondante et celui de son (dernier) conjoint, l'occupation du (dernier) conjoint.

L'inclusion parmi les facteurs socio-économiques du statut de travail de la répondante pour son occupation au moment de l'enquête ou sa plus récente occupation n'est guère justifiée. Tout au plus les auteurs affirment-ils que "de fortes interrelations entre la fécondité et le niveau de scolarité et la participation de l'épouse à l'emploi, ont été généralement observées en recherche démographique" (Rodriguez et Cleland, 1981 : 355; notre traduction). Quant à la nature de ces liens entre la participation des femmes à l'emploi et la fécondité, ils n'en disent pas plus que ce qui suit (ibid. : 345 ) :

\begin{abstract}
"L'examen des données a suggéré que la distinction la plus nette et la plus importante se trouve entre les femmes qui avaient été à l'emploi d'un non-parent et les autres. La majorité des membres du premier groupe étaient payés en espèces et travaillaient en dehors de la maison, facteurs considérés particulièrement pertinents pour l'étude de la fécondité" (traduit et souligné par nous).
\end{abstract}

C'est sur cette base que les auteurs ont pu définir le statut de travail de la répondante, à partir des trois grandes catégories suivantes : la première comprend les femmes n'ayant pas travaillé depuis leur première union; la deuxième, celles qui sont employées par un non-parent; et la troisième regroupe les travailleuses autonomes et celles qui sont employées sur la ferme familiale ou par un parent. Leur démarche analytique vise à mesurer l'effet de chaque facteur en ne tenant pas compte des autres facteurs d'une part, et après ajustement pour leurs effets, d'autre part, et cela, en contrôlant l'âge au mariage.

12. En tout, cela fait 27 populations en tenant compte de la désagrégation par ethnie dans quelques pays. 
Ils trouvent que l'effet "non ajusté" du statut de travail de la femme sur la fécondité légitime est statistiquement significatif dans 22 des 27 populations étudiées, que l'effet va dans le sens des hypothèses et que la force de l'association est considérable, surtout dans les pays de l'Amérique latine et de la Caraỉbe (Rodriguez et Cleland, $1981: 388$ ). De plus, l'effet de ce facteur demeure significatif dans 19 populations après contrôle de toutes les autres variables, les différences se trouvant à peine atténuées.

Ces résultats contrastent cependant avec ceux d'une étude des Nations Unies menée également à partir d'une analyse multivariée des données de l'EMF (United Nations, 1981). Cette étude conclut en effet que l'occupation et le statut de travail des répondantes n'auraient pas d'impact important et significatif sur l'utilisation de la contraception au moment de l'enquête, une fois leur résidence, leur éducation et le statut socio-économique de leur conjoint pris en compte. La question qui se pose alors est la suivante : comment le statut de travail des femmes pourrait-il affecter leur fécondité si ce n'est par le biais de l'utilisation de la contraception?

Face à ce paradoxe, Chidambaram et Cleland (1981) ont tenté de réconcilier ces deux séries de résultats en faisant l'hypothèse que des biais systématiques dans la déclaration des activités professionnelles surestiment l'effet du travail sur la fécondité tel que mesuré par Rodriguez et Cleland. Leur argumentation se résume ainsi : d'une part, les femmes qui viennent d'accoucher ont tendance à se retirer au moins temporairement du marché du travail; d'autre part, les femmes qui ne travaillent pas au moment de l'enquête seraient portées à ne pas déclarer leur expérience professionnelle passée, et donc à être classées comme n'ayant jamais travaillé. De ce fait, la fécondité des femmes de cette dernière catégorie s'en trouverait surestimée aux dépens de celle des femmes qui travaillent au moment de l'enquête.

Cette argumentation n'est pas vraiment convaincante pour deux raisons. En premier lieu, on peut se demander dans quelle mesure l'hypothèse d'un biais dans la déclaration de l'expérience de travail est vraisemblable et généralisable à l'ensemble des régions du Tiers-Monde. En effet, les auteurs ne citent aucun résultat pour étayer cette hypothèse. En second lieu, il faut 
également s'interroger sur la qualité des données concernant l'utilisation de la contraception au moment de l'enquête, question que ne se posent pas Chidambaram et Cleland. Pourtant, une étude récente remet sérieusement en question la validité des données concernant les principales variables intermédiaires, dont justement la contraception (Rindfuss et: alii, 1987). Dans ces circonstances, on voit mal pourquoi il faudrait privilégier une série de résultats plutôt qu'une autre, et en tirer, comme le fait notamment Cleland (1985), des conclusions définitives sur l'impact du travail sur la fécondité. Nous reviendrons sur ce point plus loin.

Deux études plus récentes des Nations Unies se sont également penchées, d'un point de vue comparatif, sur les liens entre le travail des femmes et la fécondité dans les pays en voie de développement. La première (United Nations, 1985a) utilise l'analyse de classification multiple à partir des données de 30 pays pour vérifier l'hypothèse centrale de l'approche d'incompatibilité des rôles, à savoir que les femmes travaillant à l'extérieur ("away from home") devraient avoir le plus de difficulté à concilier leur activité professionnelle et les soins aux enfants, et devraient ainsi présenter une plus faible fécondité que les autres (ibid. : 88). Le lieu de travail depuis la première union constitue donc la variable indépendante et est défini de la façon suivante : ne travaille pas; travaille à l'extérieur; travaille au foyer. Trois mesures de la fécondité sont utilisées tour à tour comme variables dépendantes : le nombre d'enfants nés vivants au cours des cinq années précédant l'enquête, la descendance atteinte au moment de l'enquête, et le nombre d'enfants désirés. L'effet de la variable indépendante sur l'une ou l'autre des variables dépendantes est ajusté selon l'âge de la répondante, son âge à la première union, son éducation, sa résidence au moment de l'enquête, et l'occupation de son (dernier) conjoint.

Les résultats obtenus sont très mitigés. Alors que dans la plupart des pays, les femmes qui n'ont pas travaillé depuis leur première union présentent la fécondité la plus élevée, la position des deux autres groupes varie et les différences en termes de fécondité ne sont guère substantielles. De plus, alors que l'association travail-fécondité est significative dans la plupart des pays, les différences de fécondité entre les trois groupes de femmes selon leur lieu de travail sont faibles, une fois prises en compte les variables contrôlées. 
La seconde étude des Nations Unies (1985b) procède à partir d'une analyse de régression multiple menée sur les données de 38 pays. Le taux de fécondité calculé comme le rapport de la descendance atteinte au nombre d'années depuis la première union, constitue la variable dépendante. La variable indépendante est l'occupation au moment de l'enquête, ou la plus récente occupation depuis la première union. Cette variable est codée en quatre catégories. La "catégorie des occupations modernes" comprend les femmes que l'EMF a codé comme professionnelles ou comme employées de bureau. Ces femmes sont généralement employées par un non-parent et travaillent à l'extérieur de la maison en échange d'un salaire ou d'une rémunération. La "catégorie de transition" regroupe les femmes qui ont été codées comme domestiques ou employées des services, et qui travaillent également pour un non-parent et à l'extérieur de la maison. Par contre, elles utilisent des qualifications traditionnelles demandant peu d'éducation (lavandière, femmes de ménages, etc.). Les femmes du "groupe mixte", c'est-à-dire les commerçantes et les ouvrières qualifiées, travaillent soit à la maison soit à l'extérieur. Travailleuses autonomes pour la plupart, elles exercent une activité qui demande un certain niveau de formation et de qualification. Enfin, la "catégorie des occupations traditionnelles" comprend les femmes qui travaillent dans l'agriculture. Ces dernières sont surtout employées par un parent, ont peu d'éducation et vivent en milieu rural (Nations Unies, 1985b : 45). Les femmes n'ayant pas travaillé depuis leur première union forment le groupe de référence.

Les hypothèses que cherche à vérifier cette étude s'inspirent à la fois de l'approche de l'incompatibilité des rôles et de celle de l'orientation des rôles de sexe. Dans ce cadre, on s'attend à ce qu'une occupation moderne soit inversement liée à la fécondité, les femmes ayant ce type d'emploi devant notamment travailler de longues heures à l'extérieur de la maison. Ce faisant, elles sont également les plus susceptibles d'être exposées aux méthodes modernes de contraception et aux valeurs associées à une famille de petite taille (Nations Unies, 1985b : 56-57). Au contraire, une occupation traditionnelle n'aurait que peu d'impact sur la fécondité, car ce type de travail est davantage compatible avec le soin aux enfants. La fécondité des femmes des secteurs "transitionnel" et "mixte" devrait se situer entre celle des deux catégories précédentes. 
Une fois prises en compte certaines caractéristiques de la répondante (durée du mariage, âge au mariage, statut au premier mariage, lieu de résidence, éducation) et de son (premier) conjoint (occupation), les résultats obtenus vont dans le même sens que les hypothèses. Mais, bien que l'occupation soit significativement associée à la fécondité dans la grande majorité des pays, les différences sont très faibles. Ainsi (Nations Unies, $1985 b$ : 45),

\begin{abstract}
"Après prise en compte des autres variables socioéconomiques, les femmes occupées dans des cccupations "modernes" se révèlent avoir un taux de fécondité impliquant un demi-enfant de moins que celui des femmes n'ayant jamais travaillé, et cela en moyenne après 20 ans de mariage" (notre traduction).
\end{abstract}

Après avoir classé les pays à partir d'un indicateur de "développement socio-économique" 13 , cette étude conclut également que la relation entre travail des femmes et fécondité est la plus forte dans les pays qui présentent un niveau élevé de développement. Dans les pays où le niveau de développement socio-économique serait le plus faible, aucune relation systématique n'est observée entre une des catégories d'occupation et la fécondité.

S'appuyant sur les résultats mitigés de ces analyses comparatives, Cleland (1985: 240) soutient que les hypothèses théoriques concernant les liens entre le travail des femmes et leur fécondité doivent être carrément abandonnées. Il conclut que la participation des femmes au "travail non familial" ne constitue ni une condition nécessaire, ni une condition suffisante au déclin de leur fécondité. Ainsi, dans l'optique de l'élaboration de futures enquêtes sur la fécondité, Cleland et alii (1987:49) estiment que :

"... (il y a) peu de justifications pour maintenir un traitement complet de l'emploi féminin dans les futurs questionnaires

13. Cet indicateur est construit à partir des données aggrégatives indépendantes de l'EMF et combine quatre variables : le produit national brut; le taux de scolarisation des garçons et des filles, primaire et secondaire confondus; le taux de mortalité infantile; les pourcentages de propriétaires d'automobile, de poste de télévision et de radio (United Nations, 1985b : 24). 
modèles. Les 17 questions dans le questionnaire de base de l'EMF devraient être réduites à quatre ou cinq, peut-être en se concentrant sur la nature de l'emploi actuel, s'il y a lieu. Les questions sur l'emploi avant le mariage devraient être considérablement limitées, parce que la relation entre cette variable et l'âge au mariage s'est révélée opiniâtrement circulaire. L'intérêt devrait être restreint à l'emploi générateur d'une rémunération en espèces ou en nature, et les tentatives infructueuses d'élargir la définition de ce qu'est le travail devraient être abandonnées. La décision de l'EMF d'inclure la mesure du travail dans le contexte de la maison ou de la ferme familiale, semble avoir été motivée plus par des considérations de justice sociale que par son effet possible sur la fécondité" (notre traduction). ${ }^{14}$

Cette position, qui revient à réduire l'information de nature socioéconomique déjà très fragmentaire dans l'EMF, s'inscrit selon nous dans le prolongement d'un long débat en démographie sociale entre les partisans d'un mode d'explication qui met l'accent sur les structures socio-économiques ${ }^{15}$, et ceux qui privilégient les déterminants culturels ${ }^{16}$. Il nous semble que les résultats des analyses comparatives examinées ici ne permettent pas de trancher ce débat. En effet, le caractère mitigé de ces résultats pourrait tout aussi bien s'expliquer par le type de données collectées par l'EMF.

En particulier, on peut s'interroger sur les concepts et leur mise en application. En effet, l'exclusion des "occupations ménagères", dans la définition du travail, ne peut être sans conséquences dans le contexte des pays en voie de développement, où ces occupations sont essentielles à la survie de la majorité des ménages. De plus, elles sont souvent étroitement imbriquées avec

14. Nous nous expliquons mal la différence entre le chiffre de 17 questions cité ici par Cleland et alii, et celui de 14 que nous retrouvons dans le tableau 1 ci-haut. Soulignons que les recommandations de Cleland et alii à l'effet de restreindre la définition du travail ont été suivies dans la dernière enquête portant sur la fécondité à Trinidad et Tobago.

15. On retrouve dans ce groupe les démographes qui ont proposé les premières formulations de la théorie de la transition démographique, Notestein (1945) et Davis (1945) notamment, de même que ceux et celles qui adoptent une approche micro-économique (e.a. Easterlin, 1983), institutionnelle (e.a. Potter, 1983) ou matérialiste (Cordell, Gregory et Piché, 1987).

16. Voir par exemple Okediji (1974), Caldwell et Caldwell (1987) et Cleland et Wilson (1987). 
les activités domestiques de production et de commercialisation. Il faut également souligner les problèmes que pose l'exercice simultané de plusieurs occupations, les enquêteurs ou enquêtrices ne devant retenir que la "principale". En ce qui concerne le lieu de travail, on retrouve les mêmes difficultés du fait qu'il revient aux enquêteurs et enquêtrices de décider si les occupations se déroulent "surtout" à l'extérieur ou à la maison. Dans ce contexte, il n'est donc guère surprenant que la qualité des données de l'EMF sur le travail ait tellement varié d'un pays à l'autre. Comme le souligne une étude des Nations Unies, "les variables du travail ont été considérées comme la source majeure d'incomparabilité des données de l'EMF" (United Nations, $1985 \mathrm{~b}: 14$; notre traduction).

Même si on ne peut pas tirer de conclusions définitives, le bilan décevant des analyses comparatives pose au moins deux questions cruciales. Premièrement, comment expliquer autrement que par la qualité des données l'absence d'associations systématiques entre le travail des femmes et leur fécondité ? Deuxièmement, si l'on persiste, comme nous le faisons, à postuler un lien important entre les deux variables, les données de l'EMF sont-elles utiles pour l'étudier? Nous proposons en conclusion quelques éléments de réponse.

\section{EN GUISE DE CONCLUSION : POUR UNE APPROCHE INSTITUTIONNELLE}

En ce qui concerne la première question, la conclusion voulant que le travail féminin n'ait pas de lien systématique avec la fécondité est à notre avis non fondée. En effet, en centrant la collecte sur l'utilisation obligatoire d'un même questionnaire de base, le programme de l'EMF devait servir en priorité à des analyses comparatives menées à partir des données d'un grand nombre de pays. Cependant, lorsque l'on considère la nature restreinte de l'information sur le travail des femmes dans le questionnaire de base de l'EMF, ce type de démarche analytique appliquée au lien entre travail des femmes et fécondité n'a de sens, selon nous, que par rapport à un cadre théorique particulier. Ce cadre théorique, dominant en démographie sociale au cours des années soixante, 
postule l'existence d'un processus uniforme et universel de changement social qui associe de façon plutôt mécanique la modernisation à l'urbanisation, à la scolarisation, au travail des femmes et à la baisse de la fécondité.

C'est dans ce cadre théorique général que s'inscrivent d'ailleurs les approches d'incompatibilité des rôles et d'orientation en termes de rôles de sexe auxquelles nous avons fait référence précédemment (Mason et Palan, 1981). Selon nous, c'est plutôt ce cadre théorique, davantage implicite que clairement exprimé dans l'EMF, qui est invalidé par les résultats décevants des analyses comparatives, et non pas comme le prétend Cleland l'existence même d'un lien entre travail des femmes et fécondité. En ce sens, certaines études de cas suggèrent que les liens entre travail féminin et fécondité existent, mais qu'ils sont trop complexes pour que l'on puisse en rendre compte à partir des approches mentionnées précédemment.

Ainsi, une étude d'Engracia (1981) menée à partir des données de l'EMF, met en évidence qu'aux Philippines les femmes les plus instruites présentent une fécondité supérieure à la moyenne, ce qui va à l'encontre de l'approche fondée sur l'orientation des rôles sexués. Cette approche postule en effet que les femmes les plus exposées aux valeurs "modernes" auront tendance à considérer le travail comme une alternative légitime au rôle de mère.

De même, une étude menée par Mason et Palan (1980) sur le travail des femmes et la fécondité en Malaisie, toujours à partir des données de l'EMF, attire l'attention sur trois séries de résultats que ne peut expliquer l'hypothèse d'incompatibilité des rôles. Premièrement, pour les femmes malaises de milieu rural, la relation entre travail et fécondité, bien que faible, est inverse alors que selon cette approche, il n'y aurait pas de raison que ces femmes cessent de travailler lorsqu'elles ont de jeunes enfants. Deuxièmement, plus que l'incompatibilité "spatio-temporelle" (c'est-à-dire la difficulté de concilier le fait d'avoir des enfants et de travailler à l'extérieur du foyer), c'est l'incompatibilité "normative" (c'est-à-dire la perception des mères quant à leur rôle) qui affecte davantage la relation entre emploi et fécondité. Enfin, pour les femmes indiennes et chinoises de milieu rural, la relation est fortement 
positive, ce que ne peut expliquer l'hypothèse de lincompatibilité, qui ne se rapporte qu'aux conditions dans lesquelles la relation négative se produit.

Pour expliquer de tels résultats, Mason et Palan proposent une approche institutionnelle plus globale qui rendrait compte de la diversité des liens entre travail féminin et fécondité. Cette approche se fonde sur le concept de structure d'opportunités des ménages ("household opportunity structure") que les auteurs définissent de la façon suivante (Mason et Palan, $1980: 38$ ) :

"Ce concept réfère aux voies de survie et de réussite économiques que les parents estiment ouvertes à eux-mêmes et à leurs enfants, voies qui influencent d'une part la division du travail à l'intérieur des familles, et d'autre part leur niveau de fécondité. Ces voies comprennent les opportunités relatives de gains monétaires que les mères ont par rapport à leurs enfants, l'importance que représentent pour le bien-être familial les rémunérations individuelles par rapport aux entreprises économiques de type coopératif, et l'importance de la scolarisation formelle pour le maintien du statut économique de la famille" (Mason et Palan, 1980 : 38; notre traduction).

Ce concept de "structure d'opportunités des ménages" est fortement apparenté à celui de "stratégies familiales de reproduction", davantage utilisé en démographie sociale (Bouchard, 1987; Woods, 1982; Simmons, 1985), et auquel nous avons recours dans nos travaux (Cordell, Gregory et Piché, 1987; Gregory et alii, 1988; Poirier, 1989). Ce concept vise à rendre compte de l'ensemble des pratiques chronologiquement articulées que les familles mettent en oeuvre pour assurer lèur reproduction. Comme le souligne Folbre (1984), ces stratégies sont élaborées dans le contexte des rapports de force qui existent à l'intérieur des ménages. Ces rapports sont à leur tour déterminés par les ensembles de contraintes et d'options qui s'offrent aux individus selon la place qu'ils occupent dans la structure sociale.

Pour illustrer cette approche, revenons à l'étude de Mason et Palan sur la Malaisie. Les auteurs soulignent que si les possibilités d'emploi salarié pour les femmes sont élevées, que le travail des enfants n'est pas rentable et que la scolarisation des enfants n'est pas un véhicule important pour l'accès aux 
ressources économiques, le travail des femmes augmentera avec la fécondité, afin d'améliorer les revenus familiaux. C'est ce qui expliquerait, par exemple, la relation positive observée pour les femmes indiennes et chinoises de milieu rural en Malaisie. Par contre, si les activités économiques sont mixtes (agriculture et commerce), que le travail des enfants est rentable, et que leur scolarisation n'est pas nécessaire, comme chez la majorité des Malais de milieu rural, les femmes travailleront, que la fécondité soit élevée ou faible. La relation sera alors faible ou inexistante. Le cas "classique" de l'incompatibilité des rôles se retrouverait lorsque l'emploi salarié est développé, le travail des enfants non rentable (ou non permis), et la scolarisation de ceux-ci obligatoire. Dans ce cas, les femmes qui travaillent auront recours à la contraception et diminueront leur fécondité, produisant ainsi une relation négative entre travail et fécondité, telle qu'observée chez les Indiennes de milieu urbain.

$\mathrm{Si}$, comme nous le croyons, cette approche institutionnelle plus globale est pertinente pour l'étude des liens entre le travail féminin et la fécondité dans les pays en développement, la question qui se pose alors est la suivante : dans quelle mesure les données de l'EMF peuvent-elles être utiles pour la mettre en application ? Avant de répondre à cette question, il convient tout d'abord de rappeler que les données de l'EMF n'ont pas été collectées dans le but premier de mener en profondeur des études de cas. C'est pourtant ce type de démarche qu'exige l'approche institutionnelle que nous avons présentée. Sur ce plan, les données de l'EMF présentent des lacunes évidentes, en raison du caractère fragmentaire de l'information socio-économique qu'elles contiennent (Entwistle et Mason, 1985). Pourtant, puisque ces données existent et que, pour beaucoup de pays en développement, elles sont les seules qui soient représentatives au niveau national, nous croyons qu'elles peuvent être utiles, mais à la condition d'être complétées par d'autres sources de données, quantitatives (recensements, enquêtes de population active, enquêtes sur les ménages) ou qualitatives (enquêtes ethnographiques). Ces sources complémentaires devraient permettre d'identifier les principales structures d'opportunités qui caractérisent une société dans un contexte historique donné, et d'en saisir les transformations.

Il est clair que cette démarche analytique est à l'opposé de ce qui s'est fait généralement jusqu'ici à partir des données de l'EMF. Cependant, nous 
croyons que cette démarche est nécessaire si nous voulons mieux comprendre la complexité des liens entre le travail féminin et la fécondité, tout en maximisant l'exploitation de données qui jusqu'à présent, à quelques exceptions près, ont donné des résultats confus, voire contradictoires.

\section{RÉFÉRENCES BIBLIOGRAPHIQUES}

BOUCHARD, G., 1987. "Sur la reproduction familiale en milieu rural : systèmes ouverts et systèmes clos." Recherches sociographiques, 28, 2-3 : 229-251.

CALDWELL, J.C. et P. CALDWELL, 1987. "The Cultural Context of High Fertility in Sub-Saharan Africa". Population and Development Review, $13,3,439-438$.

CHIDAMBARAM, V.C. et V. CLELAND, 1981. "The Contribution of the World Fertility Survey to an Understanding of Fertility Determinants and Trends". In International Population Conference. Manila 1981. Liège, IUSSP, volume no 5, 381-403.

ClELAND, J., 1985. "Marital Fertility Decline in Developing Countries : Theories and the Evidence". In J. Cleland et J. Hobcraft (édis). Reproductive Change in Developing Countries: Insights from the WFS, Oxford University Press, 223-252.

CLELAND, J. et J. HOBCRAFT (éds), 1985. Reproductive Change in Developing Countries: Insights from the WFS. Oxford University Press.

CLELAND, J. et C. SCOTT (éds), 1987. The World Fertility Survey. An Assessment. Oxford University Press.

ClELAND, J. et C. WILSON, 1987. "Demand Theories of the Fertility Transition : An Iconoclastic View". Population Studies, 41, 5-30. 
CLELAND, J. et alii, 1987. "The Core Questionnaires". In J. Cleland et C. Scott (éds). The World Fertility Survey. An Assessment. Oxford University Press, 31-75.

CORDELL, D. et J. GREGORY, 1987 (éds.). African Population and Capitalism. Historical Perspectives. London, Westview Press.

CORDELL, D., J. GREGORY et V. PICHÉ, 1987. "African Historical Demography. The Search for a Theoretical Framework." In D. Cordell et J. Gregory (éds), African Population and Capitalism. Historical Perspectives. London, Westview Press.

DAVIS, K., 1945. "The World Demographic Transition". The Annals of the American Academy of Political and Social Science, 237, 1-11.

DAVIS, K., 1987. "The World Most Expensive Survey". Sociological Forum. $2,4,829-834$.

EASTERLIN, R.A., 1983. "Modernization and Fertility : A Critical Essay". In R.A. Bulateo et R.D. Lee (éds.). Determinants of Fertility in Developing Countries, volume 2, New York, Academic Press.

ENGRACIA, L.T., 1981. Female Labor Force Participation and Fertility in the Philippines. Thèse (Ph.D.) non publiée, Logan, Utah State University.

ENTWISTLE, B. et W.M. MASON, 1985. What Has Been Learned From the World Fertility Survey About the Effects of Socio-Economic Position on Reproductive Behavior. Research Report no 85-77, Population Studies Center, University of Michigan.

FOLBRE, N., 1984. "Household Production in the Philippines : A NonClassical Approach." Economic Development and Cultural Change, 32, $2: 303-330$.

GOLDSCHEIDER, C., 1982. "Societal Change and Demographic Transition". In Chaire Quetelet 1981, Population et structures sociales. Département de démographie, Université de Louvain, Louvain-la-Neuve, Belgique, 83106.

GREGORY, J., G. NEILL, V. PICHÉ et J. POIRIER, 1988. Division du travail et structures des ménages en Guadeloupe. Montréal, Université de Montréal, Département de démographie, Rapport de recherche. 
KEMPENEERS, M., 1985. "Quand la démographie s'intéresse au travail des femmes". Cahiers québécois de démographie, 14, 2, 147-164.

KUPINSKY, S. (éd.),1977. The Fertility of Working Women : A Synthesis of International Research. New York, Praeger Publishers.

MASON, K.O. \& V.T. PALAN, 1980. Female Employment and Fertility in Peninsular Malaysia: The Maternal Role Incompatibility Hypothesis Reconsidered. Research Report, no 81-4, Population Studies Center, University of Michigan.

MASON, K.O. et V.T. PALAN, 1981. "Female Employment and Fertility in Peninsular Malaysia : the Maternal Role Incompatibility Hypothesis Reconsidered". Demography, 18, 4, 549-575.

MIRO, C.A., 1981. "The Potential of the WFS to Clarify the Socio-Economic Determinants of Fertility in Developing Countries". In WFS Conference. Records of Proceedings, volume 1, Voorburg, International Statistical Institute, 337, 363.

NEILL, G., 1988. Démographie. femmes et développement : une mise en perspective critique. Examen de synthèse, Département de démographie, Université de Montréal.

NOTESTEIN, F.W., 1945. "Population, the Long View". In E. Schultz (éd). Food for the World, University of Chicago Press, 36-57.

OKEDIJI, F.O., 1974. Changements dans le comportement individuel_en matière de procréation et valeurs culturelles. Bucarest, Union internationale pour l'étude scientifique de la population.

PARSONS, T., 1966. Societies. Evolutionary and Comparative Perspectives. Englewood Cliffs, Prentice - Hall, Inc.

POIRIER, J., 1989. Structure sociale, modes d'organisation familiale et baisse de la fécondité en Guadeloupe (1954-1982). Montréal, Université de Montréal, Département de démographie, Thèse de $\mathrm{Ph} . \mathrm{D}$.

POTTER, J., 1983. "Effects of Societal and Community Institutions on Fertility". In R.A. Bulateo et R.D. Lee (éds). Determinants of Fertility in Developing Countries, volume 2, New York, Academic Press. 
RINDFUSS, R.R. et alii, 1987. "Analyzing Birth Intervals : Implications for Demographic Theory and Data Collection". Sociological Forum, 2, 4, 811-828.

RODRIGUEZ, G. et J. CLELAND, 1981. "Socio-Economic Determinants of Marital Fertility in Twenty Countries : a Multivariate Analysis". In WFS Conference 1980: Record of Proceedings, volume 2, Voorburg, International Statistical Institute, 337-389.

SIMMONS, A., 1985. Household Demographic Strategies in Rural Latin America : Incoroorating Detailed Community Studies Into a StructuralHistorical Model. Communication présentée au Colloque Sud/Sud, organisé par l'ACELAC et l'ACEA, Université McGill, Montréal.

SINGH, S., 1984. Guyana. Jamaica and Trinidad and Tobago : SocioEconomic Differentials in Cumulative Fertility. WFS Scientific Reports, no 57.

SINGH, S., 1987. "Additions to the Core Questionnaires". In J. Cleland et C. Scott (eds), The World Fertility Survey. An Assessment, Oxford University Press, 76-92.

STANDING, G., 1983. "Women's Work Activity and Fertility". In R.A. Bulateo et R.D. Lee (eds), Determinants of Fertility in Developping Countries, volume 1, New York, Academic Press, 517-546.

TABUTIN, D., 1984. Les limites de la théorie classique de la transition démographique pour l'Occident du XIXe siècle et le Tiers-Monde actuel. Working Paper no 122, Département de démographie, Université de Louvain, Louvain-la-Neuve (Belgique).

TIPPS, D.C., 1973. "Modernization Theory and the Study of National Societies : A Critical Perspective". Comparative Studies in Society and History, 15, 2, 199-226.

UNITED NATIONS, 1981. Variations in the Incidence of Knowledge and Use of Contraception : A Comparative Analysis of World Fertility Survey Results for Twenty Developing Countries. ST/ESA/Series R/40, New York, Department of Social Affairs.

UNITED NATIONS, 1985a. World Population Trends, Population and Development Interrelations and Population Policies (1983, Monitoring Report, volume 1). Population Studies no 93, New York, Department of International Economic and Social Affairs. 
UNITED NATIONS, 1985b. Women's Employment and Fiertility A Comparative Analysis of World Fertility Survey for 38 Developing Countries. Population Studies, no 96, New York, Department of International Economic and Social Affairs.

WOODS, C.H., 1982. "Equilibrium and Historical Structural Perspectives on Migration." International Migration Review, 16, 2 : 298-319.

WFS (WORLD FERTILITY SURVEY), 1976. Questionnaires de base EMF. Documentation de base no 1 . 


\section{RÉSUMÉ - SUMMARY - RESUMEN}

POIRIER Jean, Victor PICHÉ et Ghyslaine NEILL - TRAVAIL DES FEMMES ET FÉCONDITÉ DANS LES PAYS EN DÉVELOPPEMENT : QUE NOUS A APPRIS L'ENQUETE MONDIALE DE LA FÉCONDITÉ?

L'objectif de cet article est d'examiner dans quelle mesure les données de l'Enquête mondiale de la fécondité (EMF) sont adéquates pour l'analyse de la relation entre le travail des femmes et la fécondité. Après avoir passé en revue les objectifs généraux de l'EMF, les auteurs présentent les hypothèses théoriques et les questions par lesquelles on a tenté de vérifier celles-ci. Une troisième partie est consacrée aux principales analyses comparatives réalisées, à leurs résultats fondamentaux et aux diverses interprétations qui en ont été dégagées. Les auteurs concluent en faisant ressortir en quoi ces données de l'EMF sont effectivement utiles, à la condition que l'on tienne compte du contexte institutionnel global de chaque société.

POIRIER Jean, Victor PICHÉ and Ghyslaine NEILL - FEMALE LABOR AND FERTLITY IN DEVELOPING COUNTRIES : WHAT DOES THE WORLD FERTILITY SURVEY TELL US ?

The purpose of this paper is to investigate to what extent the data of the World Fertility Survey (WFS) are useful for analysing the links between female labor and fertility. Starting with a review of the general objectives of the WFS, the authors present the theoretical expectations and the questions used for testing them. A third section is devoted to the main existing comparative studies, to the results obtained and the various interpretations to which they led. The authors conclude by assessing that the WFS data may indeed be useful, at least if due account is made of the global institutional context of each society.

POIRIER Jean, Victor PICHÉ y Ghyslaine NEILL - EL TRABAJO DE LAS MUJERES Y LA FECUNDIDAD EN LOS PAISES EN DESAROLLO : ¿ QUE NOS HA ENSENADO LA ENCUESTA MUNDIAL DE LA FECUNDIDAD?

El objetivo de este artículo es de examinar en que medida los datos de la encuesta mundial de la fecundidad (EMF) son adecuados para el análisis de la relación entre el trabajo de las mujeres y la fecundidad. Después de haber pasado revista los objetivos generales de l'EMF, los autores presentan las hipótesis teóricas y las cuestiones por las cuales se ha intentado verificar aquellas. Una tercera parte esta dedicada a los principales análisis comparativos realizados, a sus resultados fundamentales y a las diversas interpretaciones extraidas. Los autores concluyen haciendo resaltar en que estos datos de l'EMF son efectivamente útiles a la condición que se tenga en cuenta el contexto institucional global de cada sociedad. 